\title{
Mixture toxic impacts and the related mechanism of aflatoxin B1 and deoxynivalenol on embryonic zebrafish (Danio rerio)
}

\author{
Yanhua Wang ${ }^{1 \dagger}$, Qiang Wang ${ }^{1 \dagger}$, Chun $\mathrm{Ji}^{2}$, Xiaoxuan Guo ${ }^{3}$, Guiling Yang ${ }^{1}$, Dou Wang ${ }^{1}$, Hongbiao Weng ${ }^{1}$, \\ Yongzhong Qian ${ }^{3}$ and Chen Chen ${ }^{3,4^{*}}$ (i)
}

\begin{abstract}
Background: Although humans and animals are often simultaneously exposed to a variety of mycotoxins via feed and food consumption, in which aflatoxin B1 (AFB1) and deoxynivalenol (DON) is one of the most prevalent combination of mycotoxins. Many toxicological studies have merely focused on the impacts of single mycotoxins. In the present study, the combined toxicity and the related mechanism of AFB1 and DON to zebrafish (Danio rerio) were investigated.
\end{abstract}

Results: Results exhibited that DON had lower toxicity to embryonic zebrafish with a 7 -day $L C_{50}$ of $218.3 \mathrm{mg}$ a.i. $\mathrm{L}^{-1}$ in comparison to AFB1 $\left(0.031 \mathrm{mg}\right.$ a.i. $\left.\mathrm{L}^{-1}\right)$. The mixture of AFB1 and DON elicited an additive combined effect on zebrafish embryos. The levels of CAT, caspase-3, and T4 markedly varied in most single and mixture groups. The expressions of four genes (cas3, apaf-1, cc-chem, and cyp 19a) associated with oxidative stress, cellular apoptosis, immune system, and endocrine system were markedly varied upon the mixture exposure in comparison to the corresponding single exposure of AFB1 or DON.

Conclusions: Our results revealed that the impacts of a mixture could not be estimated solely based on toxicities of the single mycotoxins. Taken together, our comprehensive investigation on the mycotoxin mixtures and their potential mechanisms could better reflect the reality of mycotoxin contamination in food and feed.

Keywords: Joint toxicity, Aflatoxin B1, Deoxynivalenol, Toxic mechanism, Aquatic vertebrates

\section{Introduction}

As toxic secondary metabolites generated by a wide variety of fungi, mycotoxins can contaminate food and feed resources, which has become a worldwide problem to public health and economic significance [1]. These toxicants can cause acute toxicity or chronic disorders in animals and humans, including reduced food production

*Correspondence: chenchen02@caas.cn

†Yanhua Wang and Qiang Wang contributed equally to this article

${ }^{3}$ Key Laboratory of Agro-Product Quality and Safety of Ministry

of Agriculture, Institute of Quality Standards and Testing Technology

for Agro-Products, Chinese Academy of Agricultural Sciences,

Beijing 100081, China

Full list of author information is available at the end of the article and livestock breeding and induction of cancers and immune deficiency $[2,3]$. Most toxin-producing fungi can generate various mycotoxins simultaneously and result in the co-occurrence of multiple mycotoxins in different feed and food commodities in which aflatoxin B1 (AFB1) and deoxynivalenol (DON) is one of the most prevalent combination [4-6]. Although humans and animals can be exposed to multiple mycotoxins through the consumption of contaminated foods and feeds, the great majority of studies merely focus on the toxicology of individual mycotoxins, which might misunderstand the combined toxicities caused by the mixtures [7]. Therefore, the adverse effects of mixture mycotoxins are be growing concern and needs to be elucidated. However, 
mixture toxic effects of mycotoxins on $D$. rerio have not been well documented.

Although numerous studies have investigated the mixture effects of mycotoxins lately, most of these reports have focused on cytotoxicity testing [8]. The mixture of AFB1 and DON can decrease the viability of cells by inducing intracellular reactive oxygen species (ROS) production and promoting apoptosis in rat liver cells, which was mediated by apoptosis pathway shared between AFB1 and DON $[9,10]$. However, the cytotoxicity testing method is restricted by immortalization, limited survival, metabolic imbalance, or lack of tissue intercourse [11]. Zebrafish (Danio rerio) has been well accepted as general fish and vertebrate model to investigate the chemical toxicity and mechanism because of its easy breeding in the laboratory, great fecundity, fast development, short life cycle, and transparency of the eggs and embryos [12]. Therefore, it is an outstanding vertebrate model due to its highly developmental similarity to mammals [13]. Because of these reasons, toxicity evaluation on zebrafish can serve as a significant reference to humans [14].

Aflatoxins are a family of closely related pyranocoumarin compounds generated as secondary metabolites of the common molds Aspergillus flavus, A. parasiticus and to a lesser extent $A$. nominus [15]. As the most toxic xenobiotic and potent carcinogen, AFB1 has hepatocarcinogenic, mutagenic, and genotoxic effects [7]. Deoxynivalenol is a trichothecene, and it is mainly produced by Fusarium spp. [16]. Ingestion of DON-polluted feed can cause anorexia, and vomiting, and affect the immune system in different livestock species [17]. Since AFB1 and DON frequently occur as mixtures in various food and feed commodities, which may have additional impacts on humans in comparison to their respective compounds [18]. Up to date, the underlying mechanism of the mixture impacts in $D$. rerio remains largely unclear [19]. Thus, the hidden effects of these two mycotoxins on zebrafish embryos were investigated in the present study. Such systematic tests laid a solid foundation for future studies on the toxicological impacts and mechanism of mycotoxin mixtures on humans.

\section{Materials and methods}

\section{Materials}

AFB1 (purity $\geq 99 \%$, CAS Number: 1162-65-8) and DON (purity $\geq 99 \%$, CAS Number: $51481-10-8$ ) were purchased from Sangon Biotech (Shanghai) Co., Ltd. (China). A stock solution of AFB1 was prepared in dimethylsulfoxide (DMSO) and Tween-80, and the stock solution of DON was prepared in Milli-Q water directly. Mycotoxins of desired concentrations were prepared using reconstituted water supplemented with
$2 \mathrm{mmol} \mathrm{L}{ }^{-1} \mathrm{Ca}^{2+}, 0.5 \mathrm{mmol} \mathrm{L}{ }^{-1} \mathrm{Mg}^{2+}, 0.75 \mathrm{mmol} \mathrm{L}^{-1}$ $\mathrm{Na}^{+}$, and $0.074 \mathrm{mmol} \mathrm{L}^{-1} \mathrm{~K}^{+}[20]$.

The contents of malonaldehyde (MDA), lipid peroxidation (LPO) enzyme, antioxidant enzymes [superoxide dismutase (SOD), catalase (CAT) and peroxidase (POD)], apoptotic enzymes (caspase-3 and caspase-9), and detoxification enzymes [cytochrome P450 (CYP450), carboxylesterase (CarE) and glutathione-S-transferase (GST)], as well as the vitellogenin (VTG) and thyroid hormones (THs), such as triiodothyronine (T3) and thyroxine (T4), were determined using ELISA kits from Nanjing Jiancheng Bioengineering Institute (Nanjing, China). TransZol Up Plus RNA Kit and TransScript ${ }^{\circledR}$ II OneStep RT-PCR SuperMix were purchased from TransGen Biotech (Takara, Dalian, China). All other chemicals and compounds were of analytical grade.

\section{Maintenance and rearing conditions of fish}

Wild-type (AB strain) adult zebrafish were obtained from the China Zebrafish Research Center (Wuhan, China) and reared in a laboratory-scale polyisoprene tank (200 L) equipped with a recirculation system. The fish breeding was carried out at $27 \pm 1{ }^{\circ} \mathrm{C}$ under a photoperiod consisting of $14 \mathrm{~h}$ of light and $10 \mathrm{~h}$ of darkness. Fish were fed TetraMin Flake (fish food) complemented with brine shrimp (Artemia salina) twice a day. For egg induction, parental zebrafish (male:female ratio, 1:2) were segregated in spawning boxes (Esen Corp, Beijing, China) overnight. Light-induced spawning was carried out the next morning, and eggs were harvested within half an hour. Fertilized and morphologically normal eggs were picked for further assays. The animal study was authenticated by the Animal Care and Use Committee of Zhejiang Academy of Agricultural Sciences (Hangzhou, China).

\section{Acute toxicity determination \\ Individual mycotoxin toxicity determination}

The acute toxicity test on embryonic fish was carried out based on OECD test guideline 236 with minor modifications [21]. The median lethal concentration $\left(\mathrm{LC}_{50}\right)$ was determined using the preliminarily tested concentrations. Embryos at about $3 \mathrm{~h}$ post-fertilization (hpf) were stochastically selected and placed into 96-well plates, and each well contained one embryo and $2 \mu \mathrm{L}$ of exposure solution. Reconstituted water was employed as untreated (blank untreated) control, and solvent untreated containing the same content of DMSO and Tween-80 at the highest concentration was adopted in the AFB1 test. Each test concentration and untreated control were performed three times. Each replicate was composed of 32 wells. Embryos were challenged by six different concentrations with a geometric ratio to establish the 
concentration-mortality relationship for each mycotoxin. All 96-well plates were incubated at the conditions same as the rearing conditions. The plates were sheathed by transparent lids for 7 days. The test solution was changed every $12 \mathrm{~h}$ to maintain the concentrations of toxicants. The mortality was determined according to the signs of coagulation of embryos, lack of somite formation, non-detachment of the tail, and failure of heartbeat, and recorded after 4 and 7 days of exposure.

\section{Determination of mixture toxicity}

The mixture toxicity of mycotoxins was examined with zebrafish embryos. In this study, an equitoxic ratio was adopted to avoid that when the toxicity of a single pollutant differed greatly, one pollutant would dominate and mask the toxic effect of the other pollutant, thus changing the combined action type of mixed pollutants. Based on the $\mathrm{LC}_{50}$ values acquired in the single toxicity determination, the final concentration of the individual mycotoxin in the mixture was used as the corresponding $\mathrm{LC}_{50}$ value (equipotent combinations) to assess the mixture toxicity and type of interaction. The total concentration of each blend was methodically altered, and all the abovementioned ratios were unchanged to explore the concentration-response correlation. All experiments were performed three times for each concentration. The other test routines of mixture toxicity determination were consistent with those of the individual mycotoxin test.

\section{Subacute toxicity determination}

The subacute toxicity was determined based on the data of acute toxicity to embryos to explore the potential mechanisms of AFB1, DON, and their mixture in embryonic zebrafish.

\section{Exposure procedure}

Briefly, $1 / 320,1 / 80$, and $1 / 20$ of 7 -day $\mathrm{LC}_{50}$ for each mycotoxin were defined as the low, middle, and high concentrations, respectively. Accordingly, low, middle, and high concentrations in the combined exposure of AFB1+DON (JOT) were combinations of AFB1 and $\mathrm{DON}$ at the low, middle, and high concentrations, respectively. Besides, the blank and solvent controls were set up. About 300 embryos of $3 \mathrm{hpf}$ were stochastically picked and placed into a 1-L beaker containing $600 \mathrm{~mL}$ mycotoxin solution, and each experiment was repeated three times for each concentration. The test solution was renewed every $12 \mathrm{~h}$. After exposure for 7 days, hatched larvae were collected and rinsed twice with reconstituted water.

\section{Biochemical level determination}

Briefly, about 200 larvae were homogenized (1:20, w/v) in $50 \mathrm{mmol} \mathrm{L}^{-1}$ potassium phosphate buffer $(\mathrm{pH} 7.0)$ containing $0.5 \mathrm{mmol} \mathrm{L}^{-1} \mathrm{EDTA}$, followed by centrifugation at $12,000 \mathrm{rpm}$ for $30 \mathrm{~min}$ at $4{ }^{\circ} \mathrm{C}$. The supernatant was collected and used for the assay of biochemical parameters.

The indexes of oxidative stress, including MDA content, the activities of LPO enzyme, antioxidant enzymes including SOD, CAT, and POD, apoptosis-related enzymes including caspase- 3 and caspase-9, and detoxification enzymes including CYP450, CarE, and GST, as well as the levels of VTG and THs (T3 and T4), were determined according to the instructions of the respective assay kits.

\section{Molecular level determination}

After 7 days of exposure, approximately 30 embryos were harvested and dissolved in Trizol reagent (Takara, Dalian, China) for total RNA isolation with a previously described protocol [22]. The RNA concentration was determined at $260 \mathrm{~nm}$, and the RNA integrity was confirmed based on the absorbance ratios (OD260/OD280). Purified RNA was reversely transcribed into cDNA using a PrimeScript ${ }^{\mathrm{TM}}$ RT reagent Kit (Takara, Dalian, China). Quantitative real-time polymerase chain reaction (qRTPCR) was performed on a CFX Real-Time PCR Detection System (Bio-Rad, USA) using a SYBR Green PCR Master Mix reagent kit (Takara, Dalian, China). After an initial denaturation step at $95{ }^{\circ} \mathrm{C}$ for $30 \mathrm{~s}$, amplifications were carried out with 40 cycles at a melting temperature of $95{ }^{\circ} \mathrm{C}$ for $5 \mathrm{~s}$, and an annealing temperature of $60{ }^{\circ} \mathrm{C}$ for $30 \mathrm{~s}$. The relative expressions of the target genes were calculated using the $2^{-\Delta \Delta \mathrm{Ct}}$ method. $\beta$-actin was adopted as the internal control. All primers were designed by Primer 6.0 software and synthesized by Sangon Biotechnology (Shanghai, China) (Additional file 1: Table S1).

\section{Statistical analysis}

A probit analysis was carried out to determine the acute toxicity of mycotoxins to $D$. rerio using a previously developed program [23]. The differences between the control and exposure groups were determined using oneway analysis of variance (ANOVA), followed by Dunnett's post hoc comparison, and $P<0.05$ was considered statistically significant.

\section{Results}

Individual and mixture acute toxicity evaluations

The acute toxicity data of AFB1 and DON to embryos of D. rerio are listed in Table 1. Results exhibited that various mycotoxins had significantly different toxicities to 
Table 1 Individual and combined toxicities of aflatoxin B1 and deoxynivalenol to embryonic fish

\begin{tabular}{|c|c|c|c|c|c|}
\hline \multirow[t]{2}{*}{ Exposure time (d) } & \multicolumn{2}{|c|}{$\mathrm{LC}_{50}(95 \% \mathrm{FL})^{\mathrm{a}} \mathrm{mg}$ a.i. $\mathrm{L}^{-1}$} & \multicolumn{2}{|c|}{$\mathrm{LC}_{50}(95 \% \mathrm{FL})^{\mathrm{b}} \mathrm{mg}$ a.i. $\mathrm{L}^{-1}$} & \multirow[t]{2}{*}{$\mathrm{Al}^{\mathrm{C}}$ value } \\
\hline & Aflatoxin B1 & Deoxynivalenol & Aflatoxin B1 & Deoxynivalenol & \\
\hline 4 & $0.087(0.065-0.12)$ & $>1000$ & $--^{d}$ & - & - \\
\hline 7 & $0.031(0.017-0.042)$ & $218(164-301)$ & $0.018(0.013-0.021)$ & $126(91-147)$ & -0.16 \\
\hline
\end{tabular}

fish. At a 4-day interval, AFB1 exerted higher toxicity to D. rerio than DON. At a 7-day interval, AFB1 still elicited higher toxicity to zebrafish than DON. The toxicity of AFB1 was 7042 times higher in comparison to DON after exposure for 7 days. Moreover, the toxicities of AFB1 and DON were significantly elevated when the exposure period was extended. To understand the interaction between different mycotoxins in the mixture toxicity against $D$. rerio, we detected the $\mathrm{LC}_{50}$ values of $\mathrm{AFB} 1$ and DON in their mixture after exposure for 7 days. According to the $\mathrm{LC}_{50}$ values of AFB1 and DON individually and in the mixture, the mixture toxicity of AFB1 and DON displayed an additive effect on $D$. rerio.

\section{Biochemical level determination Oxidative stress determination}

The MDA level in the high-concentration group of AFB1 and the low-concentration group of DON was surprisingly inhibited in comparison to the control group. Besides, a markedly lower level of MDA was also detected in the middle-concentration group of JOT in comparison to the corresponding single-exposure groups of AFB1 and DON (Fig. 1A). The LPO activity was radically increased in the high-concentration group of AFB1. By contrast, the activity was markedly elevated in the low-concentration group of DON. Moreover, its activity was also radically decreased in the middle-concentration group of JOT in comparison to the corresponding singleexposure group of DON (Fig. 1B). The SOD activity was not outstandingly altered in all the single and JOT exposure groups. Additionally, no outstanding alteration was monitored in all the JOT exposure groups in comparison to the corresponding single-exposure groups of AFB1 or DON (Fig. 1C). The CAT activity remained unchanged in all the single-exposure groups. However, the activity was markedly decreased in the high-concentration group of JOT in comparison to the corresponding singleexposure group of DON (Fig. 1D). The POD activity was diminished in the low-concentration group of DON in comparison to the control group. On the contrary, the activity was remarkably increased in the low-concentration group of JOT in comparison to the corresponding single DON exposure group (Fig. 1E).

\section{Apoptosis and detoxification enzyme activities}

The activity of caspase-3 was prominently induced in the middle-concentration group of AFB1 in comparison to the control group. Besides, its activity was prominently induced in the low-concentration group of JOT in comparison to the corresponding single-exposure group of DON (Fig. 2A). Similarly, the caspase-9 activity was not noteworthily varied in all the single and JOT exposure groups. Besides, its activity was not markedly changed in all the JOT exposure groups in comparison to the corresponding single-exposure groups of AFB1 or DON (Fig. 2B). The CYP450 activity was conspicuously reduced in the high-concentration group of AFB1. In contrast, its activity was conspicuously increased in the high-concentration group of JOT in comparison to the corresponding single AFB1 group (Fig. 2C). Similar to the CAT activity, the CarE activity was not observably different in all the single-exposure groups in comparison to the control group. However, the level was observably elevated in the high-concentration JOT group in comparison to the corresponding single DON group (Fig. 2D). The GST activity was extraordinarily enhanced in the low-concentration groups of AFB1 and JOT, and the high-concentration group of DON. By contrast, its activity was extraordinarily inhibited in the high-concentration JOT group in comparison to the corresponding single DON group (Fig. 2E).

\section{TH and VTG levels}

The T3 level was manifestly reduced in the low-concentration group of DON in comparison to the control group and the corresponding JOT exposure group. Contrarily, its level was manifestly elevated in the highconcentration group of DON in comparison to the control group (Fig. 3A). The T4 level was enormously lessened in the low-concentration group of DON and 

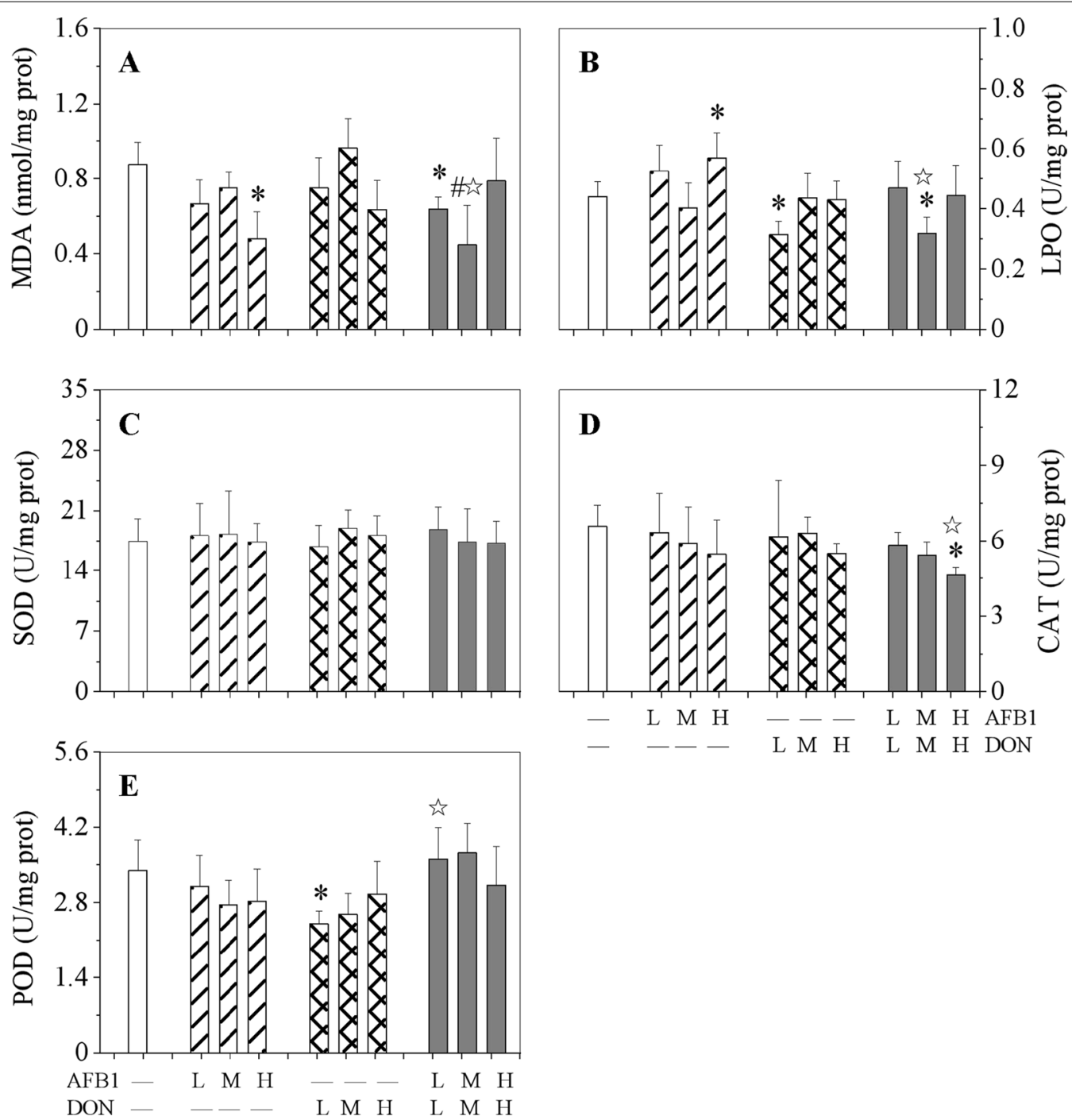

Fig. 1 The oxidative reactions of fish embryos administrated with AFB1, DON and their combinations. Data are presented as the means \pm standard deviation of three triplicates. ${ }^{*} p<0.05$, substantial alteration by comparison with the control; ${ }^{\#} p<0.05$, substantial alteration by comparison with the AFB1 administration group at counterpart concentration; ${ }^{\text {* }} p<0.05$, substantial alteration by comparison with the DON administration group at counterpart concentration. AFB1 aflatoxin B1, DON deoxynivalenol, L low concentration, $M$ middle concentration, $H$ high concentration

the high-concentration group of AFB1 in comparison to the control group. Nonetheless, its level was enormously increased in the middle- and high-concentration groups of JOT in comparison to the corresponding single-exposure groups of AFB1 (Fig. 3B). The VTG level was drastically decreased in the middle-concentration groups of AFB1 and DON in comparison to the control groups. Conversely, its level was drastically elevated in the high-concentration group of JOT in comparison to the control group (Fig. 3C).

\section{Determinations of gene expression} Influence on genes involved in anti-oxidative stress and cell apoptosis

The expression level of $\mathrm{Mn}$-sod was markedly increased in the middle-concentration groups of DON and JOT, as well as in the high-concentration groups of AFB1 and DON. However, its expression was sharply decreased in the high-concentration group of JOT in comparison to the corresponding single AFB1 group (Fig. 4A). The expression of $\mathrm{Cu} / \mathrm{Zn}$-sod was remarkably elevated 

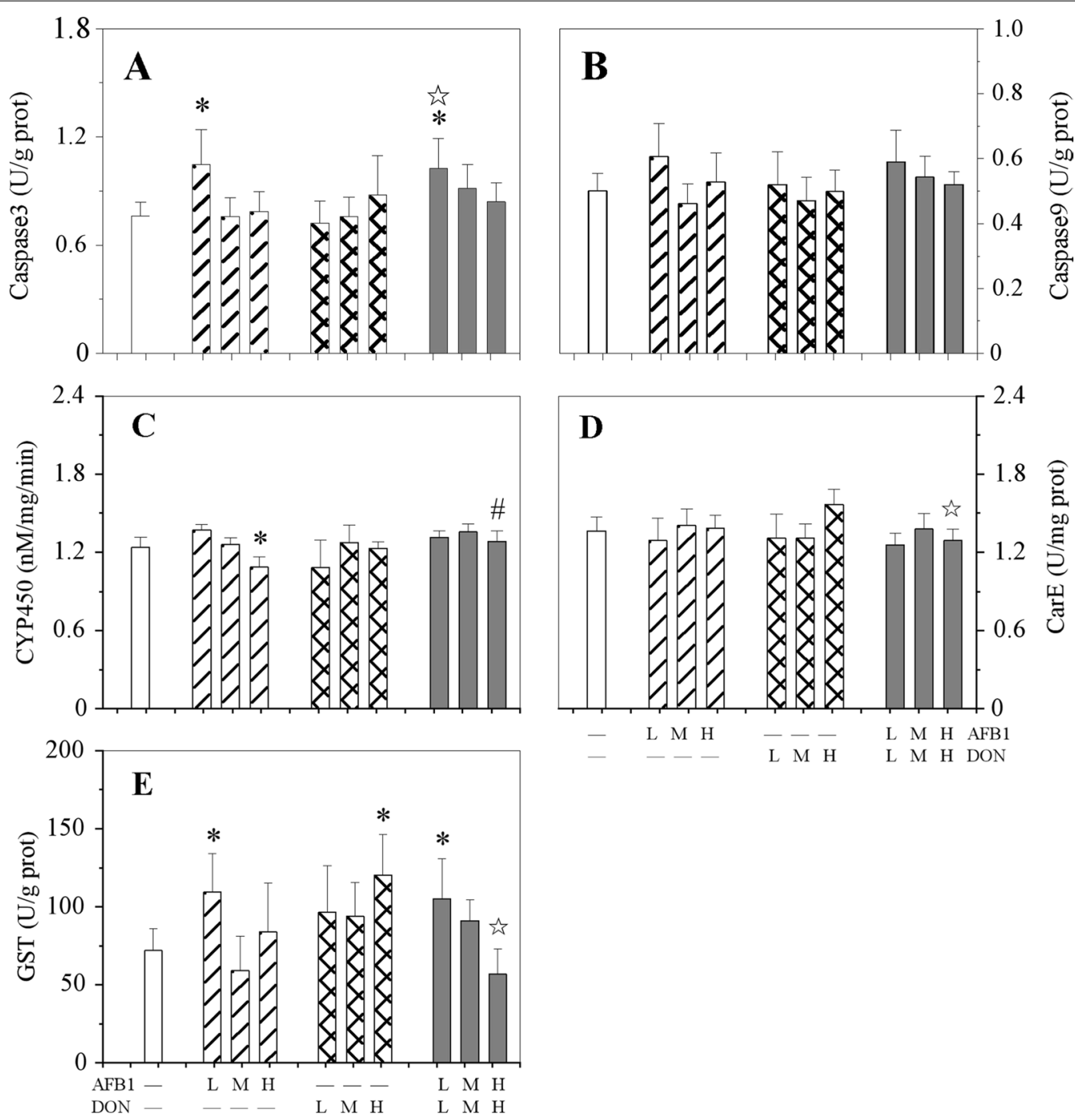

Fig. 2 The apoptotic and detoxification enzyme activities of fish embryos administrated with AFB1, DON and their combinations. Data are presented as the means \pm standard deviation of three triplicates. ${ }^{*} p<0.05$, substantial alteration by comparison with the control; ${ }^{\#} p<0.05$, substantial alteration by comparison with the AFB1 administration group at counterpart concentration; $p<0.05$, substantial alteration by comparison with the DON administration group at counterpart concentration. AFB1 aflatoxin B1, DON deoxynivalenol, $L$ low concentration, $M$ middle concentration, $H$ high concentration

in the middle-concentration groups of AFB1 and DON in comparison to the control group. Additionally, the level of expression was remarkably induced in the highconcentration group of AFB1 (Fig. 4B). The gpx expression was substantially elevated in the low-concentration group of DON in comparison to the corresponding JOT group (Fig. 4C). The cas3 expression was considerably elevated in the middle-concentration groups of AFB1 and DON. However, its expression was considerably reduced in the high-concentration group of JOT in comparison to the corresponding single AFB1 group (Fig. 4D). The expression of cas 9 was surprisingly increased in the high-concentration groups of AFB1 and JOT. Furthermore, its expression was surprisingly elevated in the low-concentration group of JOT (Fig. 4E). The apaf-1 expression was radically reduced in the low-concentration group of DON. Furthermore, the apaf- 1 expression was also radically reduced in the middle-concentration group of JOT in comparison to the corresponding single DON group (Fig. 4F). The expression of bax was outstandingly accelerated in all the single-exposure groups of AFB1 and DON. Similar to the Cu/Zn-sod expression, the expression of $b a x$ was not altered in all the JOT exposure groups in comparison to the corresponding single 


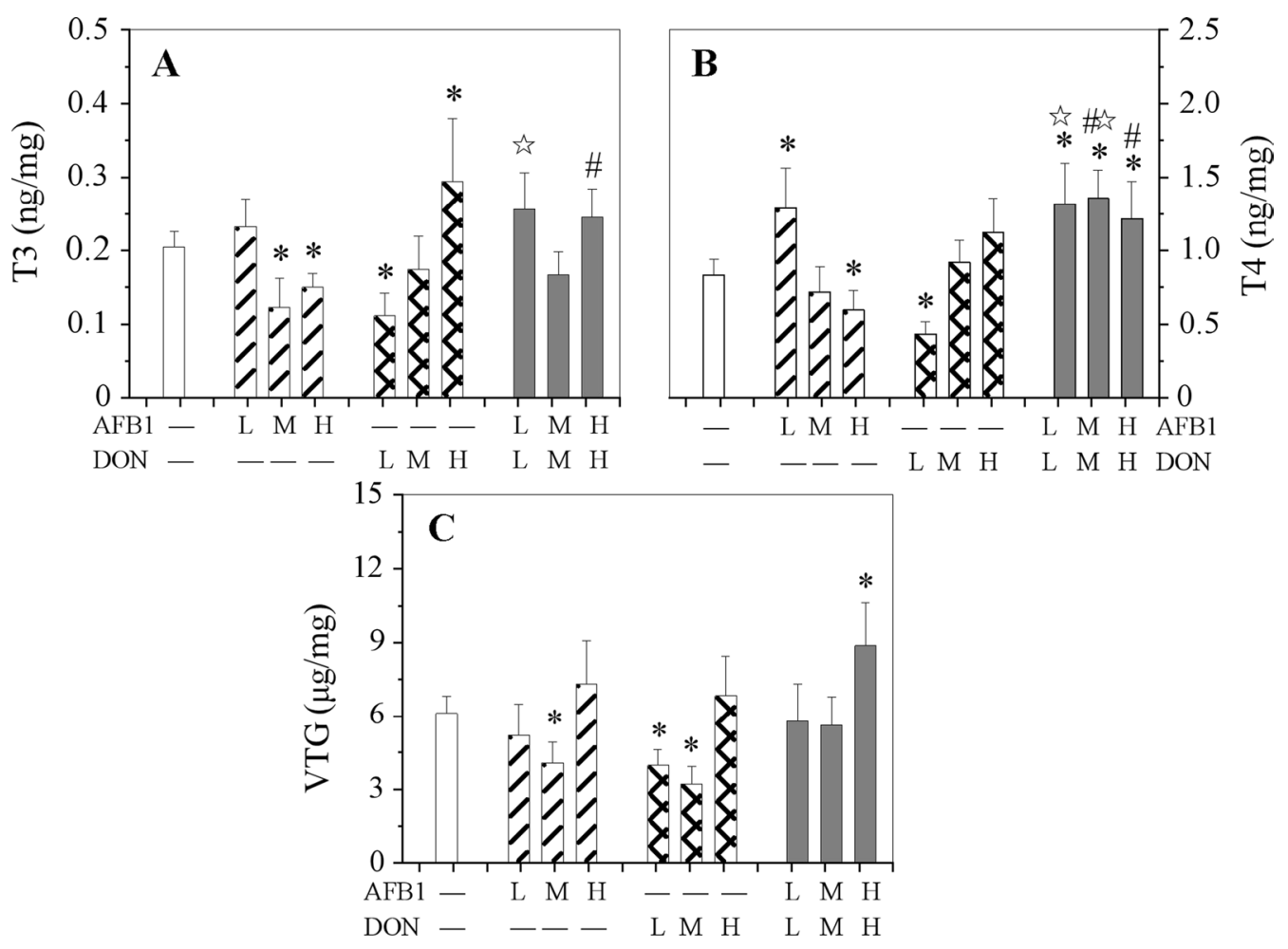

Fig. 3 Thyroid hormones and VTG levels of fish embryos administrated with AFB1, DON and their combinations. Data are presented as the means \pm standard deviation of three triplicates. ${ }^{*} p<0.05$, substantial alteration by comparison with the control; $\# p<0.05$, substantial alteration by comparison with the AFB1 administration group at counterpart concentration; ${ }^{*} p<0.05$, substantial alteration by comparison with the DON administration group at counterpart concentration. AFB1 aflatoxin B1, DON deoxynivalenol, $L$ low concentration, $M$ middle concentration, $H$ high concentration

AFB1 or DON group (Fig. 4G). The expression of $p 53$ was markedly elevated in the middle- and high-concentration groups of AFB1. Similar to the expressions of $\mathrm{Cu} / \mathrm{Zn}$-sod and $b a x$, the expression of $p 53$ did not change in all the JOT exposure groups in comparison to the corresponding single-exposure group of AFB1 or DON (Fig. 4H).

\section{Influence on genes involved in immunology system and hypothalamic-pituitary-thyroid (HPT) axis}

The $I L-8$ expression was enhanced in the middleconcentration group of AFB1 in comparison to the corresponding JOT exposure group. Moreover, its expression was also increased in the high-concentration group of DON (Fig. 5A). The expression of cc-chem was prominently increased in the low-concentration group of JOT in comparison to the corresponding DON exposure group. Furthermore, the expression level was prominently enhanced in the high-concentration group of JOT in comparison to the corresponding single-exposure group of AFB1 or DON (Fig. 5B). The cxcl-clc expression was noteworthily up-regulated in the middle- and high-concentration groups of DON.
Also, its expression was noteworthy up-regulated in the high-concentration group of JOT in comparison to the control group and the corresponding single-exposure group of AFB1 (Fig. 5C). The expression of TR $\alpha$ was conspicuously elevated in the low-concentration groups of DON and JOT. Nonetheless, its expression was not conspicuously altered in all the JOT exposure groups in comparison to the corresponding single-exposure group of AFB1 or DON (Fig. 5D). The expression of tsh was observably induced in the high-concentration groups of AFB1 and DON. Similar to the TR $\alpha$ expression, the expression of $t s h$ did not observably change in all the JOT exposure groups when compared with the corresponding single AFB1 or DON exposure groups (Fig. 5E). The expression of dio1 was extraordinarily increased in the low-concentration groups of AFB1 and DON. Moreover, its expression was extraordinarily elevated in the middle-concentration group of JOT in comparison to the individual AFB1 exposure group. Contrarily, the expression of dio1 was extraordinarily reduced in the middle-concentration group of AFB1 (Fig. 5F). 

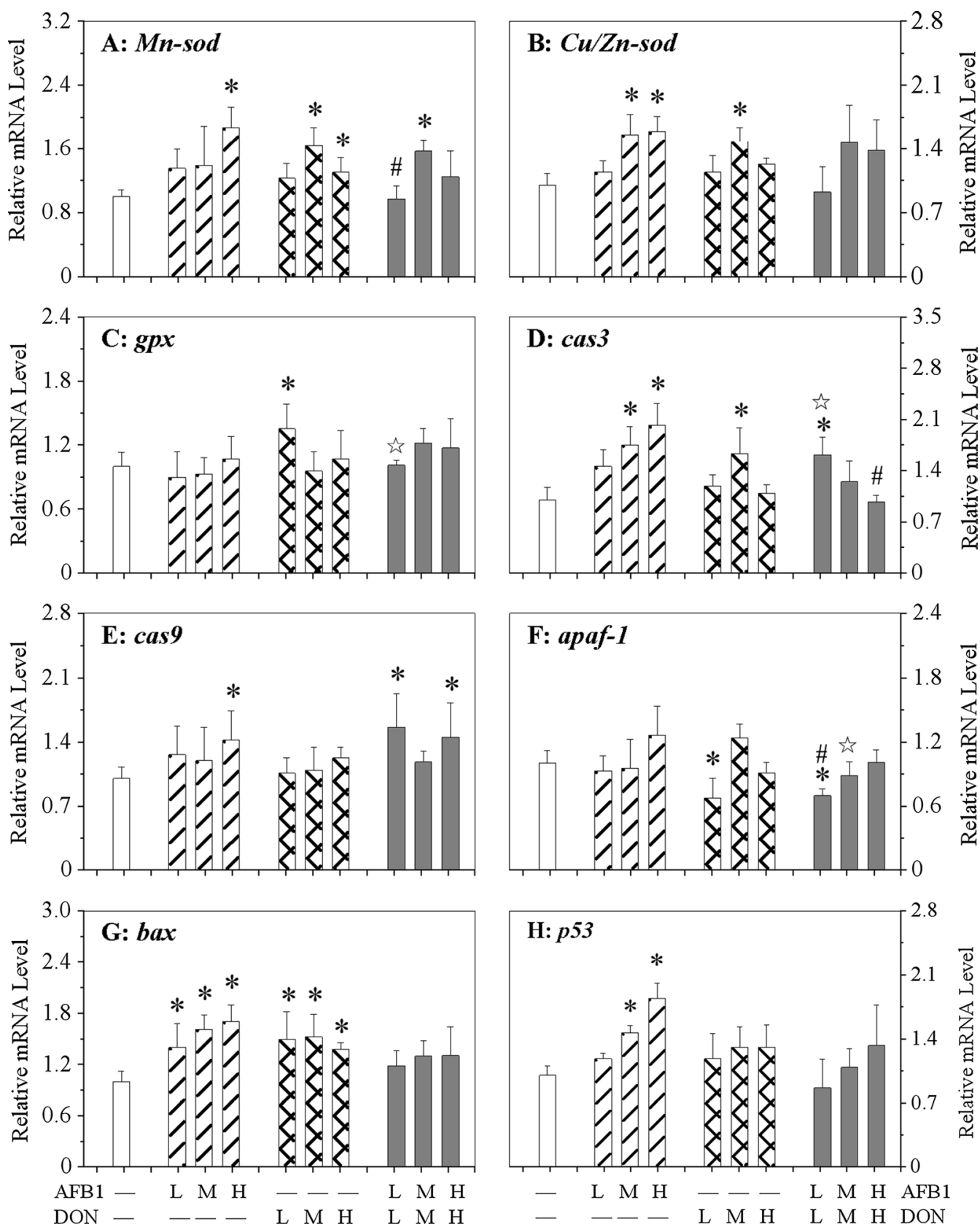

Fig. 4 Influences on transcription levels of genes associated with the anti-oxidative systems and cell apoptosis of fish embryos administrated with AFB1, DON and their combinations. Data are presented as the means \pm standard deviation of three triplicates. ${ }^{*} p<0.05$, substantial alteration by comparison with the control; $\# p<0.05$, substantial alteration by comparison with the AFB1 administration group at counterpart concentration; " $p<0.05$, substantial alteration by comparison with the DON administration group at counterpart concentration. AFB1 aflatoxin B1, DON deoxynivalenol, $L$ low concentration, $M$ middle concentration, $H$ high concentration

Influence on genes involved in hypothalamic-pituitarygonadal (HPG) and hypothalamic-pituitary-adrenal (HPA) axes

Similar to the SOD and caspase-9, the ER $\alpha$ and $E R \beta 1$ expressions were not manifestly different in all the single and JOT exposure groups in comparison to the control groups. Additionally, their expressions were not manifestly different in the JOT exposure groups in comparison to their corresponding single-exposure groups (Fig. 6A, B). The expression of cyp 17 was enormously enhanced in all the AFB1 and JOT exposure groups (except for the high-concentration groups of AFB1 and JOT). Moreover, 

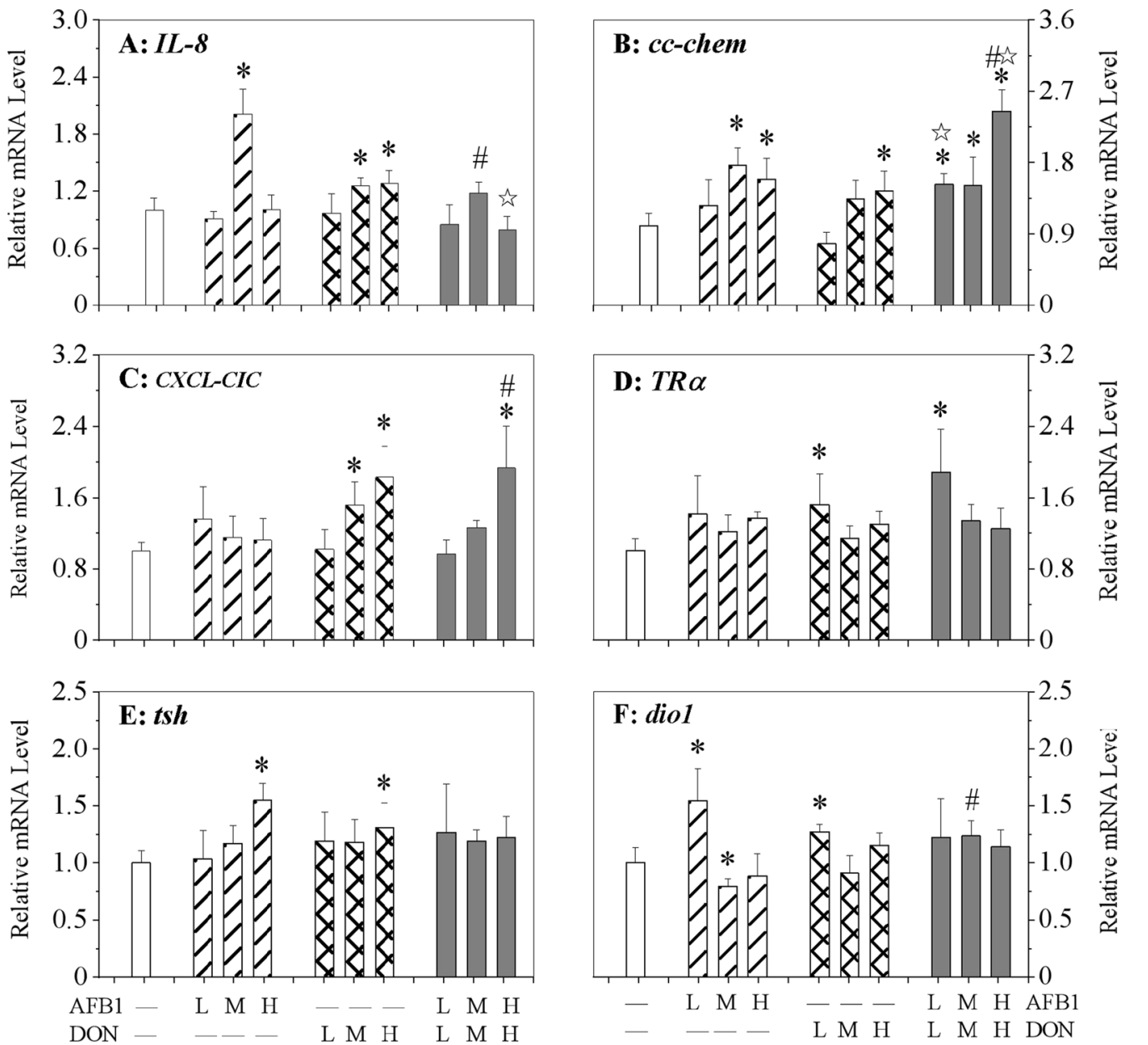

Fig. 5 Influences on transcription levels of genes associated with immunology system and HPT axis of fish embryos administrated with AFB1, DON and their combinations. Data are presented as the means \pm standard deviation of three triplicates. ${ }^{*} p<0.05$, substantial alteration by comparison with the control; $\#<<0.05$, substantial alteration by comparison with the AFB1 administration group at counterpart concentration; ${ }^{*} p<0.05$, substantial alteration by comparison with the DON administration group at counterpart concentration AFB1 aflatoxin B1, DON deoxynivalenol, L low concentration, $\mathrm{M}$ middle concentration, $H$ high concentration

its expression was enormously enhanced in the middleconcentration group of DON (Fig. 6C). The expression of cyp19a was not drastically altered in all the singleexposure groups. Nevertheless, its expression was markedly elevated in the low-concentration group of JOT in comparison to the control group and the corresponding single-exposure group of AFB1 or DON. The cyp19a expression was also remarkably induced in the middleconcentration group of JOT in comparison to the control group (Fig. 6D). The crh expression was sharply elevated in the high-concentration group of AFB1 in comparison to the control group. On the contrary, its expression was sharply decreased in the low-concentration group of JOT (Fig. 6E). The expression of $g r$ was markedly elevated in the high-concentration groups of AFB1 and JOT. Its expression was remarkably up-regulated in the middleconcentration group of AFB1 in comparison to the corresponding JOT exposure group. In contrast, its expression was markedly suppressed in the low-concentration group of JOT (Fig. 6F).

\section{Integrated biomarker response (IBR)}

To uncover toxic difference of AFB1, DON and their mixtures with different concentrations, IBR was used to integrate all the determined indicators. Data of zebrafish embryos exposed to mycotoxins at different concentrations were selected to calculate IBR index. Toxicity order of the individual and mixture mycotoxins at different concentration can be compared. The results were represented as star plot (Fig. 7). Overall, AFB1 and JOT 

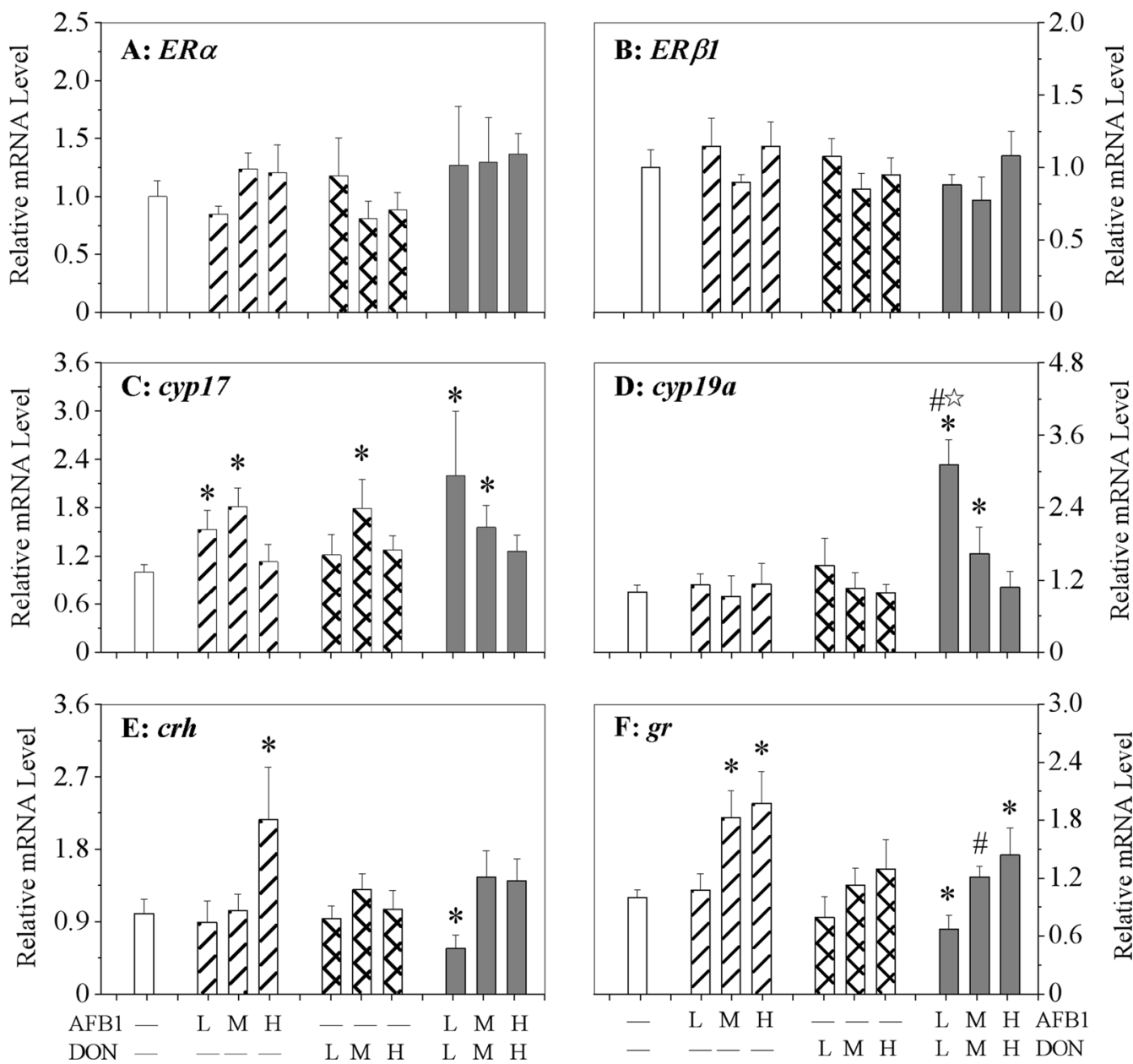

Fig. 6 Influences on transcription levels of genes associated with the HPG and HPA axes of fish embryos administrated with AFB1, DON and their combinations. Data are presented as the means \pm standard deviation of three triplicates. ${ }^{*} p<0.05$, substantial alteration by comparison with the control; $" \#<0.05$, substantial alteration by comparison with the AFB1 administration group at counterpart concentration; ${ }^{*} p<0.05$, substantial alteration by comparison with the DON administration group at counterpart concentration. AFB1 aflatoxin B1, DON deoxynivalenol, $L$ low concentration, $\mathrm{M}$ middle concentration, $H$ high concentration

exposures exhibited stronger response than DON to the antioxidant biomarkers. In contrast, AFB1 and DON elicited the strongest response among all the exposures to apoptotic and detoxification biomarkers, respectively (Fig. 7B, C). Besides, the JOT exposure displayed the strongest response among all the exposures to the endocrine system and immune system biomarkers (Fig. 7D, E).

\section{Discussion}

Acute toxicity test provides useful information on a wide range of concentrations that can be adopted in the following toxicity testing and the assessment of the therapeutic impacts of toxins [24]. Former reports have displayed that the 4-day $\mathrm{LC}_{50}$ value of AFB1 to zebrafish larvae is $0.51 \mathrm{mg}$ a.i. $\mathrm{L}^{-1}$, which is not consistent with our findings [25]. Such a prominent difference might be likely attributed to the different tested life periods. Moreover, our data showed that AFB1 possessed stronger toxicity to zebrafish in comparison to DON. The determined mycotoxins had prominently different toxicities, which might be attributed to deviations in toxicokinetics and dynamics, such as uptakes, supersession, excretion, or binding to the target sites in $D$. rerio [26]. Therefore, the exposure of AFB1 and DON had latent risks to aquatic animals. Nevertheless, previous investigations on AFB1 and DON have mainly focused on their single toxic effect, while the toxicity of their combinations remains largely unknown.

Because of the natural co-occurrence in the food of mycotoxin, a great deal of attention has been paid to the effects of the mixture exposure [27]. Zebrafish toxicity 

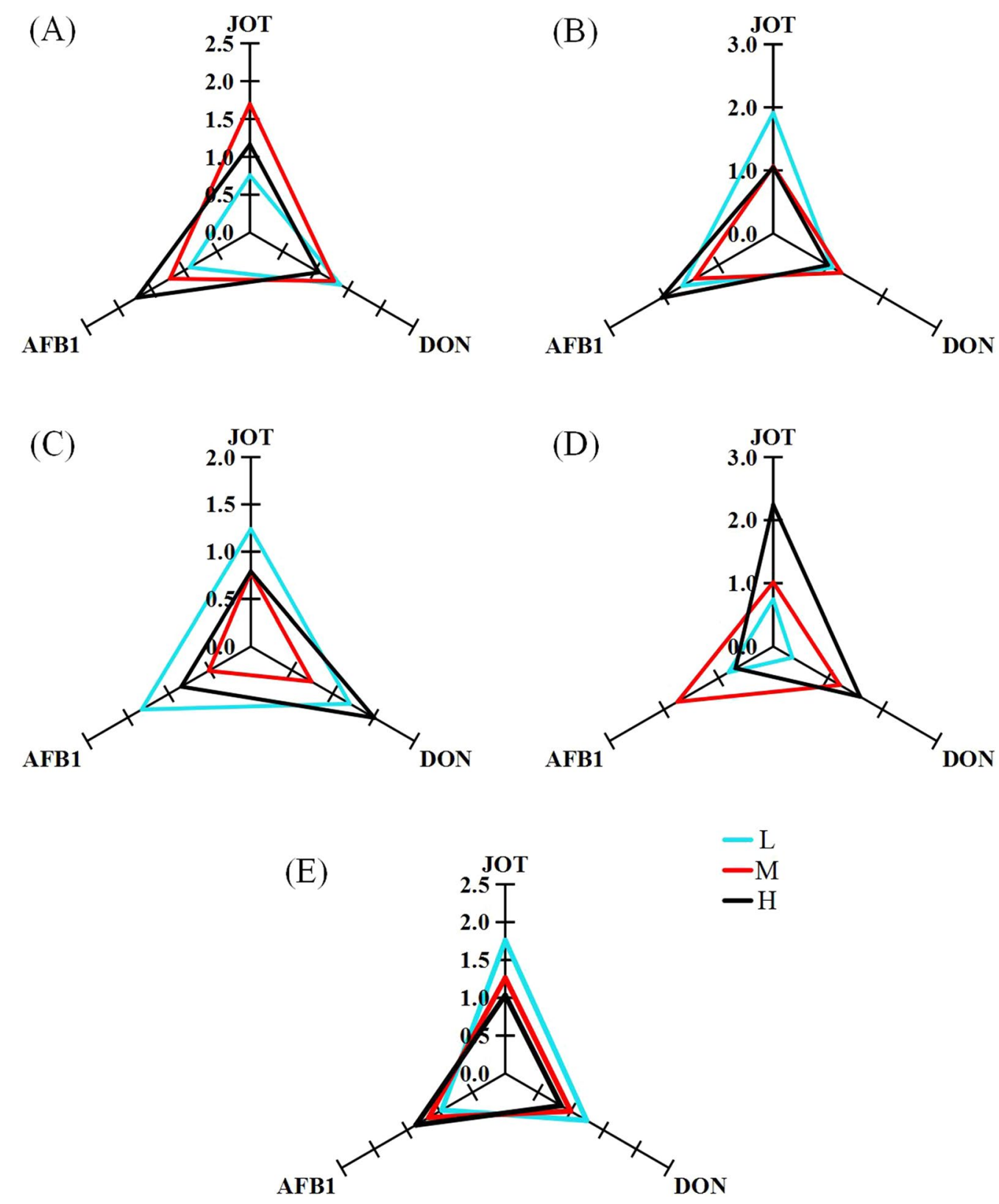

Fig. 7 Star plots of integrated biomarker response of different parameters. A IBR index of antioxidant biomarkers; B IBR index of apoptotic biomarkers; C IBR index of detoxification biomarkers; D IBR index of endocrine system biomarkers; $\mathbf{E}$ IBR index of immune system biomarkers. AFB 1 aflatoxin B1, DON deoxynivalenol, JOT joint treatment of AFB1 and DON, L low concentration, $M$ middle concentration, $H$ high concentration

studies can serve as an important reference to humans [28-31]. Consequently, embryonic zebrafish were adopted to evaluate the mixture toxic effect of AFB1 and DON in this study. An additive effect was observed by exposing zebrafish embryos to AFB1 and DON mixtures, indicating that the mixture of AFB1 and DON had stronger mixture toxicity in comparison to their single compounds [19]. Our findings manifested that it was imminently essential to assess the combined toxicity of mycotoxins because the toxicity evaluation of mycotoxins is generally performed barely on single compounds, which might result in a misleading toxic effect in realistic situations.

With the wide existence of mycotoxins in food, it is very necessary to clarify the detoxification mechanism of mycotoxins in humans and how mycotoxins impair 
and influence human's detoxification mechanism [32]. Our findings indicated that changed CYP 450 and GST activities might contribute to the toxic action of AFB1 in D. rerio, and the elevated GST activity might be the toxic mechanism of DON in the animals. Furthermore, the decreased CarE and GST activities in the high-concentration group of JOT in comparison to the corresponding single-exposure group of DON, and the enhanced CYP450 activity in the high-concentration group of JOT might result in the overall additive toxicity of AFB1 and DON [33]. Therefore, all the determined three detoxification enzymes were involved in the detoxification mechanism of mixture toxicity.

As a cell suicide mechanism, apoptosis can get rid of superfluous or undesired cells [34]. The caspase family has a critical function in cell apoptosis, and caspase- 3 has been shown as an important executor that is activated downstream in apoptosis pathways [35]. As a transcription factor, the P53 protein can prevent tumorigenesis via inducing cell cycle arrest and apoptosis [36]. Apaf-1 is the molecular core of the apoptosome, which is the executioner of mitochondria-dependent apoptosis [37]. Caspase- 9 can bind to the apoptosome where it can induce executioner caspases, such as caspase-3 [38]. As a cardinal proapoptotic member of the BCL-2 family, the BCL2 -associated $\mathrm{X}$ protein $(b a x)$ can mediate the critical equilibrium in a cell cycle [39]. The present study exerted that the caspase- 3 activity was markedly induced in the AFB1 exposure, while no prominent change in caspase-9 was observed, which was not in line with the gene expression pattern. AFB1 apparently stimulated the expressions of cas3, cas9, bax, and P53 at the mRNA level, showing that this mycotoxin caused apoptosis via activating P53, which directly triggered the expression of gene encoding the proapoptotic protein $(b a x)$, then induced the critical executor of apoptosis (cas3) activation and subsequently resulted in cell apoptosis [35]. Moreover, the expressions of cas 3 , cas 9 , and apaf- 1 were markedly altered in the JOT group, implying that regular apoptosis was influenced by the co-exposure of AFB1 and DON.

Environmental stressors can immediately stimulate oxidative stress via a complex physiological process, and oxidative stress occurs when the level of reactive oxygen species (e.g., $\mathrm{O}_{2}^{-}, \mathrm{H}_{2} \mathrm{O}_{2}, \mathrm{OH}$, etc.) is higher than the scavenging activities of antioxidants $[40,41]$. SOD can scavenge $\mathrm{O}_{2}{ }^{-}$and catalyze it to $\mathrm{O}_{2}$ and $\mathrm{H}_{2} \mathrm{O}_{2}$ [34]. The $\mathrm{H}_{2} \mathrm{O}_{2}$ generated is further detoxified via CAT and other enzymes, such as POD. The changes in their contents may indicate the extent of damage to the organism [42]. Both SOD and CAT activities in the single mycotoxin exposure groups were not markedly altered, indicating that the determined concentration of single mycotoxin was insufficient to induce prominent oxidative stress. However, a prominent reduction was found in the high-concentration group of JOT in comparison to the corresponding single-exposure group of DON, which could be attributed to an overwhelmed antioxidant capacity [43]. MDA is the primary by-product of LPO, and an elevation in its content incarnates the degree of the cell damage caused by free radicals [44]. However, the MDA level was reduced in the AFB1 and JOT exposure groups, potentially suggesting that LPO was reduced by antioxidants. The expressions of anti-oxidative genes are necessary for evaluating antioxidant capacity [35]. In our current study, AFB1 and DON exposures up-regulated the expressions of $\mathrm{Mn}$ sod and $\mathrm{Cu} / \mathrm{Zn}$-sod at the mRNA level, which was not consistent with the SOD activity, suggesting that SOD was primarily modulated at the protein level. The increased expressions of $\mathrm{Mn}$-sod and $\mathrm{Cu} / \mathrm{Zn}$-sod may induce the SOD activity and eliminate the superoxide anion radical caused by the exposure of AFB1 and DON. Besides, the up-regulation of $g p x$ at the mRNA level could also activate the gpx activity. Such discrepancy between antioxidant activity and gene expression might also be attributed to the presence of multiple gene copies in the species, a time-lag, and post-transcriptional modifications [45].

THs play an important role in the growth and development of fish and the thyroid endocrine system, while the HPT axis can regulate the thyroid system [46]. In the current study, the levels of T3 and T4 were markedly changed after exposure to AFB1 or DON, and their contents were also distinctly altered in the JOT exposure groups. Besides, the T4 level was markedly increased in the JOT exposure. The expression of the TR $\alpha$ gene was dramatically enhanced in the low-concentration group of DON, indicating that a mechanism negatively responded to the reduced T4 level [47]. On the contrary, a marked up-regulation of the TR $\alpha$ gene was found in the low-concentration group of JOT, implying that there was a positive feedback mechanism because of the induced T4 level [48]. Noticeably increased expression of $t s h$ was found in the high-concentration groups of AFB1 and DON in comparison to the control group. Iodothyronine deiodinases (Dio) possess an important function in modulating the circulation of peripheral TH levels and maintaining the ratios of T4 and T3 in vertebrates [49]. Dio1 affects iodine recovery and TH degradation. The expression of dio1 was negatively associated with T3 and T4 levels in fish upon exposure to AFB1, DON, and their mixture. Consequently, the changes in $\mathrm{T} 3$ and $\mathrm{T} 4$ contents in zebrafish indicate the maladjustment of THs [50]. The expressions of thyroid-associated genes were also considerably changed, indicating that AFB1, DON, and their mixture were thyroid disruptors in embryonic zebrafish. 
VTG is considered a biomarker of estrogen-associated endocrine disorders, and the generation of VTG is regulated via $17 \beta$-estradiol activation of estrogen receptors (ERs) [51, 52]. The VTG content was obviously reduced in the AFB1 and DON exposure groups, indicating that the two mycotoxins had the antiestrogenic effect. Conversely, the VTG content was increased in the JOT exposure group, implying that the mixture of AFB1 and DON possessed the estrogenic effect [53]. HPG axis modulates sex hormones, which are tightly associated with procreation in fish [54]. Surprisingly up-regulated expressions of cyp 17 and cyp $19 a$ were found in the AFB1, DON, or JOT group. Besides, the cyp $19 a$ expression was remarkably up-regulated in the low-concentration group of JOT implies that AFB1, $\mathrm{DON}$, and their mixture could impair the reproduction of zebrafish.

The main function of the HPA axis is to modulate the adaptive stress response of organisms [55]. The expressions of $\mathrm{crh}$ and $g r$ were noticeably varied in most of AFB1 and JOT exposure groups, implying that AFB1 and its combination with DON could impact the development of zebrafish. Environmental pollutants can elevate or reduce the expressions of immune-related genes [56]. Our present study exhibited that the single and JOT exposures increased the expressions of $I L$ 8, cc-chem, and cxcl-cic at the mRNA level. This finding might imply that latent tissue damage triggered by AFB1, DON, and their blend provoked the up-regulation of the investigated cytokines in this study [57]. Moreover, AFB1, DON, and their mixture might impair the immune function and elevate the susceptivity to toxicants for fish.

A test of multiple toxicities in zebrafish is helpful in comprehensively understanding the toxic mechanisms of environmental pollutants [36, 52]. To comprehensively assess the mixture mechanisms of AFB1 and DON in fish, we attempted to assess the toxic effects of mixture mycotoxins on oxidative stress, immunotoxicity, cell apoptosis, and endocrine system in the early developmental stage of zebrafish at various endpoints. Our data provided solid evidence on the mixture toxic mechanism of AFB1 and DON in aquatic organisms. In the present study, the altered enzyme activity reflected the extent of cellular damage. Nevertheless, different biochemical enzymes have different sensitivity to mycotoxins. To validate this hypothesis, further study is still required to evaluate the changes of these enzymes at the protein level. The zebrafish can adapt to various stimuli induced by exogenous mycotoxins via a potential mechanism by up/down-regulating genes. Therefore, it is indispensable to evaluate the mechanism underlying the different alternations in immune-associated genes.

\section{Conclusions}

AFB1 exhibited greater acute toxicity than DON to the embryos of $D$. rerio and the mixture presented an additive effect. The levels of CAT, caspase-3, and T4 were markedly varied in most single and mixture groups. The expression levels of four genes (cas3, apaf-1, cc-chem, and cyp19a) associated with oxidative stress, cellular apoptosis, immune system, and endocrine system varied markedly in the mixture group in comparison to the corresponding single group. A comprehensive assessment of toxic effects of AFB1, DON, and their mixture using multiple endpoints offered valuable insights into the total toxicity induced by single and mixture mycotoxins in zebrafish as well as its potential mechanism.

\section{Abbreviations \\ AFB1: Aflatoxin B1; DON: Deoxynivalenol; ROS: Reactive oxygen species; MDA: Malonaldehyde; LPO: Lipid peroxidation; SOD: Superoxide dismutase; CAT: Catalase; POD: Peroxidase; CYP450: Cytochrome P450; CarE: Carboxylesterase; GST: Glutathione-S-transferase; VTG: Vitellogenin; THs: Thyroid hormones; T3: Triiodothyronine; T4: Thyroxine; $\mathrm{LC}_{50}$ : Median lethal concentration; JOT: The combined exposure of AFB1 and DON; L: Low concentration; M: Middle concentration; $\mathrm{H}$ : High concentration.}

\section{Supplementary Information}

The online version contains supplementary material available at https://doi. org/10.1186/s12302-021-00529-4.

Additional file 1: Table S1. Gene primer sequences in real time quantitative $P C R$ reaction.

\section{Acknowledgements}

The authors acknowledge the technical assistance of Siyi Fang and Hailiang Guo (Zhejiang Academy of Agricultural Sciences).

\section{Authors' contributions}

YW: conceptualization, data curation, formal analysis, writing —original draft, writing - review and editing. QW: conceptualization, data curation, formal analysis, writing —original draft, project administration. CJ: conceptualization, investigation, methodology, resources. XG: conceptualization, investigation, methodology, resources. GY: conceptualization, data curation, formal analysis, writing —original draft. DW: writing —original draft, investigation, methodology, resources. HW: supervision, funding acquisition. YQ: conceptualization, supervision, funding acquisition, and project administration. CC: conceptualization, writing —original draft, supervision, funding acquisition, project administration, writing — review and editing. All authors read and approved the final manuscript.

\section{Funding}

The research was supported by the National Key Research and Development Program of China [Grant Number 2018YFC1603104], the National Natural Science Foundation of China [Grant Number 31771894] and Shanghai Agriculture Applied Technology Development Program, China [Grant Number 2021, NO.3-2].

Availability of data and materials Not applicable. 


\section{Declarations}

Ethics approval and consent to participate

Not applicable.

\section{Consent for publication}

Not applicable.

\section{Competing interests}

The authors declare that they have no competing interests.

\section{Author details}

${ }^{1}$ State Key Laboratory for Managing Biotic and Chemical Threats to the Quality and Safety of Agro-Products, Institute of Quality and Standard for Agro-Products, Zhejiang Academy of Agricultural Sciences, Hangzhou 310021,

Zhejiang, China. ${ }^{2}$ Tongzhou District Comprehensive Food Inspection Center, Nantong 226300, Jiangsu, China. ${ }^{3}$ Key Laboratory of Agro-Product Quality and Safety of Ministry of Agriculture, Institute of Quality Standards and Testing Technology for Agro-Products, Chinese Academy of Agricultural Sciences, Beijing 100081, China. ${ }^{4}$ School of Public Health, Shandong University, Jinan 250012, Shandong, China.

Received: 19 May 2021 Accepted: 13 July 2021

Published online: 27 July 2021

\section{References}

1. Liew WP, Mohd-Redzwan S (2018) Mycotoxin: its impact on gut health and microbiota. Front Cell Infect Microbiol 8:60

2. Adegbeye MJ, Reddy PRK, Chilaka CA, Balogun OB, Elghandour MMMY, Rivas-Caceres RR, Salem AZM (2020) Mycotoxin toxicity and residue in animal products: prevalence, consumer exposure and reduction strategies-a review. Toxicon 177:96-108

3. Yang C, Song G, Lim W (2020) Effects of mycotoxin-contaminated feed on farm animals. J Hazard Mater 389:122087

4. Wallin S, Gambacorta L, Kotova N, Lemming EW, Nälsén C, Solfrizzo M, Olsen M (2015) Biomonitoring of concurrent mycotoxin exposure among adults in Sweden through urinary multi-biomarker analysis. Food Chem Toxicol 83:133-139

5. Ma R, Zhang L, Liu M, Su YT, Xie WM, Zhang NY, Dai JF, Wang Y, Rajput SA, Qi DS, Karrow NA, Sun LH (2018) Individual and combined occurrence of mycotoxins in feed ingredients and complete feeds in China. Toxins 10(3):113

6. De Ruyck K, Huybrechts I, Yang S, Arcella D, Claeys L, Abbeddou S, De Keyzer W, De Vries J, Ocke M, Ruprich J, De Boevre M, De Saeger S (2020) Mycotoxin exposure assessments in a multi-center European validation study by 24-hour dietary recall and biological fluid sampling. Environ Int 137:105539

7. Rushing BR, Selim MI (2019) Aflatoxin B1: a review on metabolism, toxicity, occurrence in food, occupational exposure, and detoxification methods. Food Chem Toxicol 124:81-100

8. Smith MC, Gheux A, Coton M, Madec S, Hymery N, Coton E (2018) In vitro co-culture models to evaluate acute cytotoxicity of individual and combined mycotoxin exposures on Caco-2, THP-1 and HepaRG human cell lines. Chem Biol Interact 281:51-59

9. Sun LH, Lei MY, Zhang NY, Gao X, Li C, Krumm CS, Qi DS (2015) Individual and combined cytotoxic effects of aflatoxin B1, zearalenone, deoxynivalenol and fumonisin B1 on BRL 3A rat liver cells. Toxicon 95:6-12

10. Zhou H, George S, Hay C, Lee J, Qian H, Sun X (2017) Individual and combined effects of aflatoxin B1, deoxynivalenol and zearalenone on HepG2 and RAW 264.7 cell lines. Food Chem Toxicol 103:18-27

11. Sun H, Wang Y, Cheff DM, Hall MD, Shen M (2020) Predictive models for estimating cytotoxicity on the basis of chemical structures. Bioorg Med Chem 28(10):115422

12. Bambino K, Chu J (2017) Zebrafish in toxicology and environmental health. Curr Top Dev Biol 124:331-367

13. Shen C, Zuo Z (2020) Zebrafish (Danio rerio) as an excellent vertebrate model for the development, reproductive, cardiovascular, and neural and ocular development toxicity study of hazardous chemicals. Environ Sci Pollut Res 27(35):43599-43614

14. Lomba L, Ribate MP, Zuriaga E, García CB, Giner B (2019) Acute and subacute effects of drugs in embryos of Danio rerio. QSAR grouping and modelling. Ecotoxicol Environ Saf 172:232-239

15. Caceres I, Khoury AA, Khoury RE, Lorber S, Oswald IP, Khoury AE, Atoui A, Puel O, Bailly JD (2020) Aflatoxin biosynthesis and genetic regulation: a review. Toxins 12(3):150

16. Urbanek KA, Habrowska-Górczyńska DE, Kowalska K, Stańczyk A Domińska K, Piastowska-Ciesielska AW (2018) Deoxynivalenol as potential modulator of human steroidogenesis. J Appl Toxicol 38(12):1450-1459

17. Male D, Wu W, Mitchell NJ, Bursian S, Pestka JJ, Wu F (2016) Modeling the emetic potencies of food-borne trichothecenes by benchmark dose methodology. Food Chem Toxicol 4:178-185

18. Alassane-Kpembi I, Schatzmayr G, Taranu I, Marin D, Puel O, Oswald IP (2017) Mycotoxins co-contamination: methodological aspects and biological relevance of combined toxicity studies. Crit Rev Food Sci Nutr 57(16):3489-3507

19. Gruber-Dorninger C, Jenkins T, Schatzmayr G (2019) Global mycotoxin occurrence in feed: a ten-year survey. Toxins 11(7):375

20. ISO (1996) Water quality-Determination of the acute lethal toxicity of substances to a freshwater fish Brachydanio rerio Hamilton-Buchanan (Teleostei, Cyprinidae) — part 3: flow-through method. ISO 7346-3

21. OECD (2013) OECD guidelines for the testing of chemicals, Fish Embryo Acute Toxicity (FET) test. OECD, Paris, France. No. 236

22. Teng M, Zhu W, Wang D, Yan J, Qi S, Song M, Wang C (2018) Acute exposure of zebrafish embryo (Danio rerio) to flutolanil reveals its developmental mechanism of toxicity via disrupting the thyroid system and metabolism. Environ Pollut 242(Pt B):1157-1165

23. Chi H (1997) Computer program for the probit analysis. National Chung Hsing University, Taichung

24. Vidal A, Mengelers M, Yang S, De Saeger S, De Boevre M (2018) Mycotoxin biomarkers of exposure: a comprehensive review. Compr Rev Food Sci Food Saf 17(5):1127-1155

25. Cullen JM, Newberne PM (1994) Acute hepatotoxicity of aflatoxins. In: Eaton DL, Groopman JD (eds) The toxicology of aflatoxins-human health, veterinary and agricultural significance. Academic Press, San Diego, pp 3-26

26. Schrenk D, Gao L, Lin G, Mahony C, Mulder PPJ, Peijnenburg A, Pfuhler S, Rietjens IMCM, Rutz L, Steinhoff B, These A (2020) Pyrrolizidine alkaloids in food and phytomedicine: occurrence, exposure, toxicity, mechanisms, and risk assessment-a review. Food Chem Toxicol 136:111107

27. Reisinger N, Schürer-Waldheim S, Mayer E, Debevere S, Antonissen G, Sulyok M, NagI V (2019) Mycotoxin occurrence in maize silage-a neglected risk for bovine gut health? Toxins 11(10):577

28. Juan-García A, Bind MA, Engert F (2020) Larval zebrafish as an in vitro model for evaluating toxicological effects of mycotoxins. Ecotoxicol Environ Saf 202:110909

29. Domijan AM, Marjanović Čermak AM, Vulić A, Tartaro Bujak I, Pavičić I, Pleadin J, Markov K, Mihaljević B (2019) Cytotoxicity of gamma irradiated aflatoxin B and ochratoxin A. J Environ Sci Health B 54(3):155-162

30. du Plessis B, Regnier T, Combrinck S, Heinrich P, Braunbeck T (2019) Effect of $\mathrm{pH}$ on the toxicity of fumonisins towards the RTL-W1 cell line and zebrafish (Danio rerio) embryos. Toxicol Lett 313:101-107

31. Zhou H, George S, Li C, Gurusamy S, Sun X, Gong Z, Qian H (2017) Combined toxicity of prevalent mycotoxins studied in fish cell line and zebrafish larvae revealed that type of interactions is dose-dependent. Aquat Toxicol 193:60-71

32. Lyagin I, Efremenko E (2019) Enzymes for detoxification of various mycotoxins: origins and mechanisms of catalytic action. Molecules 24(13):2362

33. Jin $Y$, Gao Y, Zhang H, Wang L, Yang K, Dong H (2020) Detoxification enzymes associated with butene-fipronil resistance in Epacromius coerulipes. Pest Manag Sci 76(1):227-235

34. Kang R, Li R, Dai P, Li Z, Li Y, Li C (2019) Deoxynivalenol induced apoptosis and inflammation of IPEC-J2 cells by promoting ROS production. Environ Pollut 251:689-698

35. Yang F, Li L, Chen K, Li C, Wang Y, Wang G (2019) Melatonin alleviates $\beta$-zearalenol and HT-2 toxin-induced apoptosis and oxidative stress in bovine ovarian granulosa cells. Environ Toxicol Pharmacol 68:52-60

36. Ben Salah-Abbès J, Belgacem H, Ezzdini K, Abdel-Wahhab MA, Abbès S (2020) Zearalenone nephrotoxicity: DNA fragmentation, apoptotic gene 
expression and oxidative stress protected by Lactobacillus plantarum MON03. Toxicon 175:28-35

37. Omur AD, Yildirim B, Saglam YS, Comakli S, Ozkaraca M (2019) Activity of resveratrol on the influence of aflatoxin B1 on the testes of Sprague dawley rats. Pol J Vet Sci 22(2):313-320

38. Chen J, Yang S, Huang S, Yan R, Wang M, Chen S, Cai J, Long M, Li P (2020) Transcriptome study reveals apoptosis of porcine kidney cells induced by fumonisin B1 via TNF signalling pathway. Food Chem Toxicol 139:111274

39. Wang J, Jin Y, Wu S, Yu H, Zhao Y, Fang H, Shen J, Zhou C, Fu Y, Li R, Wang R, Wang J, Zheng K, Fan Q, Chen B, Zhang J (2019) Deoxynivalenol induces oxidative stress, inflammatory response and apoptosis in bovine mammary epithelial cells. J Anim Physiol Anim Nutr 103(6):1663-1674

40. Agahi F, Álvarez-Ortega N, Font G, Juan-García A, Juan C (2020) Oxidative stress, glutathione, and gene expression as key indicators in SH-SY5Y cells exposed to zearalenone metabolites and beauvericin. Toxicol Lett 334:44-52

41. Taroncher M, Pigni MC, Diana MN, Juan-García A, Ruiz MJ (2020) Does low concentration mycotoxin exposure induce toxicity in HepG2 cells through oxidative stress? Toxicol Mech Methods 30(6):417-426

42. Rašić D, Micek V, Klarić MS, Peraica M (2019) Oxidative stress as a mechanism of combined OTA and CTN toxicity in rat plasma, liver and kidney. Hum Exp Toxicol 38(4):434-445

43. Marček T, Vuletić MV, Španić V (2018) Biochemical changes in ears of wheat genotypes subjected to Fusarium spp. attack. Acta Biol Hung 69(4):493-504

44. Egresi A, Süle K, Szentmihályi K, Blázovics A, Fehér E, Hagymási K, Fébel $H$ (2020) Impact of milk thistle (Silybum marianum) on the mycotoxin caused redox-homeostasis imbalance of ducks liver. Toxicon 187:181-187

45. Zhao W, Wang L, Liu M, Jiang K, Wang M, Yang G, Qi C, Wang B (2017) Transcriptome, antioxidant enzyme activity and histopathology analysis of hepatopancreas from the white shrimp Litopenaeus vannamei fed with aflatoxin B1(AFB1). Dev Comp Immunol 74:69-81

46. Parsons AE, Lange A, Hutchinson TH, Miyagawa S, Iguchi T, Kudoh T, Tyler CR (2020) Expression dynamics of genes in the hypothalamic-pituitarythyroid (HPT) cascade and their responses to 3,3',5-triiodo-L-thyronine (T3) highlights potential vulnerability to thyroid-disrupting chemicals in zebrafish (Danio rerio) embryo-larvae. Aquat Toxicol 225:105547

47. Parsons A, Lange A, Hutchinson TH, Miyagawa S, Iguchi T, Kudoh T, Tyler CR (2019) Molecular mechanisms and tissue targets of brominated flame retardants, BDE-47 and TBBPA, in embryo-larval life stages of zebrafish (Danio rerio). Aquat Toxicol 209:99-112

48. Gogoi P, Kalita JC (2020) Effects of butylparaben exposure on thyroid peroxidase (TPO) and type 1 iodothyronine deiodinase (D1) in female Wistar rats. Toxicology 443:152562

49. Xu C, Niu L, Guo H, Sun X, Chen L, Tu W, Dai Q, Ye J, Liu W, Liu J (2019) Long-term exposure to the non-steroidal anti-inflammatory drug (NSAID) naproxen causes thyroid disruption in zebrafish at environmentally relevant concentrations. Sci Total Environ 676:387-395

50. Spaan K, Haigis AC, Weiss J, Legradi J (2019) Effects of 25 thyroid hormone disruptors on zebrafish embryos: a literature review of potential biomarkers. Sci Total Environ 656:1238-1249

51. Fuentes N, Silveyra P (2019) Estrogen receptor signaling mechanisms. Adv Protein Chem Struct Biol 116:135-170

52. Mukherjee U, Samanta A, Biswas S, Das S, Ghosh S, Mandal DK, Maitra S (2020) Bisphenol A-induced oxidative stress, hepatotoxicity and altered estrogen receptor expression in Labeo bata: impact on metabolic homeostasis and inflammatory response. Ecotoxicol Environ Saf 202:110944

53. Tran TKA, Yu RMK, Islam R, Nguyen THT, Bui TLH, Kong RYC, O'Connor WA, Leusch FDL, Andrew-Priestley M, MacFarlane GR (2019) The utility of vitellogenin as a biomarker of estrogenic endocrine disrupting chemicals in molluscs. Environ Pollut 248:1067-1078

54. Corton JC, Kleinstreuer NC, Judson RS (2019) Identification of potential endocrine disrupting chemicals using gene expression biomarkers. Toxicol Appl Pharmacol 380:114683

55. Luo Y, Chen H, Li D, Zhan M, Hou L, Dong W, Luo Y, Xie L (2020) The effects of norethindrone on the ontogeny of gene expression along the hypothalamic-pituitary-adrenal and hypothalamic-pituitary-gonadal axes in zebrafish (Danio rerio). Sci Total Environ 747:141554

56. Torraca V, Mostowy S (2018) Zebrafish infection: from pathogenesis to cell biology. Trends Cell Biol 28(2):143-156

57. Iwanami N, Hess I, Schorpp M, Boehm T (2017) Studying the adaptive immune system in zebrafish by transplantation of hematopoietic precursor cells. Methods Cell Biol 138:151-161

\section{Publisher's Note}

Springer Nature remains neutral with regard to jurisdictional claims in published maps and institutional affiliations.

\section{Submit your manuscript to a SpringerOpen ${ }^{\odot}$ journal and benefit from:}

- Convenient online submission

- Rigorous peer review

- Open access: articles freely available online

- High visibility within the field

- Retaining the copyright to your article

Submit your next manuscript at $\boldsymbol{\nabla}$ springeropen.com 